\title{
Factors influencing home discharge after inpatient rehabilitation of older patients: a systematic review
}

\author{
Irma H. J. Everink ${ }^{1 *}$, Jolanda C. M. van Haastregt ${ }^{1 *}$, Sofie J. M. van Hoof ${ }^{1}$, Jos M. G. A. Schols ${ }^{1,2}$ \\ and Gertrudis I. J. M. Kempen ${ }^{1}$
}

\begin{abstract}
Background: Although rehabilitation for older patients has the potential to improve function and prevent admission to nursing homes, returning home after discharge is not possible for all patients. Better understanding of patient factors related to discharge home may lead to more realistic rehabilitation goals, more targeted rehabilitation interventions and better preparation of both patient and informal caregiver for discharge. Various studies provided insight into factors related to home discharge after stroke rehabilitation, but we still lack insight into factors related to home discharge in non-stroke patients. Therefore, the aim of this review is to provide an overview of factors influencing home discharge in older non-stroke patients admitted to an inpatient rehabilitation unit.

Methods: A systematic literature search was executed in the databases PubMed, EMBASE, CINAHL and Web of Science to retrieve articles published between January 2000 and October 2015. The search focused on factors related to home discharge after rehabilitation for older patients. Studies were included if home discharge after rehabilitation was assessed as an outcome measure and if the non-stroke population was, on average, 65 years or older and admitted to an inpatient rehabilitation unit.

Results: Eighteen studies were included. The methodological quality was moderate to good in 15 studies. The factors significantly associated with home discharge are younger age, non-white ethnicity, being married, better functional and cognitive status, and the absence of depression.

Conclusions: Because various factors are significantly associated with home discharge of older non-stroke patients after rehabilitation, we recommend assessing these factors at admission to the rehabilitation unit. Further research into the factors that lack sufficient evidence concerning their association with home discharge is recommended.
\end{abstract}

Keywords: Aged, Geriatrics, Rehabilitation, Patient discharge

\section{Background}

Hospitalization among older adults often results in functional decline and deterioration in self-care abilities [1]. Hospital stay is associated with inactivity and immobility, and prolonged hospital stay may have harmful effects such as muscle weakness, contractures and atrophy [2]. This impedes many community-dwelling older persons to return home directly after hospital discharge, especially frail

\footnotetext{
*Correspondence: i.everink@maastrichtuniversity.nl; j.vanhaastregt@ maastrichtuniversity.nl

${ }^{1}$ Department of Health Services Research, Faculty of Health, Medicine and Life Sciences and CAHPRI School for Public Health and Primary care, Maastricht University, Maastricht, The Netherlands

Full list of author information is available at the end of the article
}

patients with comorbidity and no family caregivers. In such cases, patients may be temporarily admitted to an inpatient rehabilitation unit. Such units use a multidisciplinary and comprehensive set of evaluative, diagnostic and therapeutic interventions focused on restoring functional capacity, activities of daily living and cognitive function $[3,4]$. A study by Bachmann and colleagues revealed that rehabilitation among older patients has the potential to improve function, prevent permanent admission to nursing homes, and to decrease mortality [5].

Returning home is considered an indicator of successful rehabilitation and is frequently used as an indicator of quality of care $[6,7]$. Yet, several studies have shown 
that a considerable number of older patients cannot return to their initial living arrangement after discharge from a rehabilitation unit, and have to be admitted to long-term care facilities [8-10].

Gaining more insight into the patient characteristics (measured at admission) related to returning to the initial living arrangement, may help care professionals to set more realistic rehabilitation goals and to prepare patients and informal caregivers for probable changes in their living arrangement after discharge [11]. Furthermore, increased insight into factors related to returning home may result in more accurate referrals to follow-up care after hospital discharge and therefore in a more efficient allocation of resources $[5,12,13,14]$.

In recent years, a substantial number of studies have been carried out to identify prognostic factors of home discharge after stroke rehabilitation [15-18]. Factors frequently found to be related to non-home discharge in stroke patients were older age, lower level of activities of daily living (ADL) functioning, the presence of cognitive disturbances and gender [15]. However, inpatient rehabilitation is also recommended for older patients with other medical conditions, such as those with Parkinson's disease, amputation, arthritis, orthopedic disorders, chronic cardiac and pulmonary disease, and major multiple trauma. There is still a lack of insight into factors related to home discharge among this heterogeneous group of patients who often suffer from various comorbidities that influence the clinical course of their rehabilitation trajectory [19]. In contrast to stroke patients, non-stroke patients are more likely to be medically unstable: they are often admitted to the rehabilitation unit after trauma or an exacerbation of their illness and their rehabilitation trajectory is often complex. A better understanding in the factors related to home discharge might lead to establishing more realistic rehabilitation goals, tailored rehabilitation treatment, and a better preparation of patients and informal caregivers for the transition back home. Therefore, the purpose of the present study was to provide an overview of the factors influencing home discharge in older non-stroke patients admitted to an inpatient rehabilitation unit.

\section{Methods}

\section{Search strategy}

On the 15th of October 2015, a systematic search in four electronic databases (PubMed, EMBASE, CINAHL and Web of Science) was conducted. The search was focused on studies written in English published between 01-012000 and 15-10-2015. This timeframe was chosen to provide a realistic overview of the current situation in rehabilitation care for geriatric patients. Search terms used for the search strategy were the type of care, 'rehabilitation', combined with the Boolean operator 'AND' with search terms related to the rehabilitation setting ("rehabilitation unit" OR "rehabilitation center" OR "rehabilitation centre" OR "geriatric postacute rehabilitation" OR "geriatric postacute rehabilitation" OR "intermediate care facilities" OR "skilled nursing facilities" OR "rehabilitation department" OR "inpatient rehabilitation" OR "department of rehabilitation" OR "rehabilitation ward"), the population ("aged"), the outcome measure ("discharge location" OR "living arrangements" OR "living setting" OR "independent living" OR "discharge destination" OR "home discharge" OR "community discharge") and the focus of the research question ("determinant" $\mathrm{OR}$ "prognos"” OR "indicator" OR "influenc" OR "predict"* OR "correlat" OR "relat"*" OR "prognosis" OR "associat"”). The full search strategy can be found in an additional file [see Additional file 1]. Additional studies were located based on the reference lists of the included studies.

\section{Study selection}

Studies had to meet the following inclusion criteria:

- patients with a mean (or if not provided, a median) age of 65 years or older, who were admitted to an inpatient rehabilitation unit;

- factors potentially influencing discharge destination of these patients were measured within a week after admission to the rehabilitation unit;

- discharge location (home discharge versus non home discharge) was assessed as an outcome measure.

All studies that included patients who suffered from stroke were excluded from the review, also if the stroke patients only constituted a part of the study population. Furthermore, studies that only focused on a medical diagnosis as an influencing factor of home discharge were excluded from this review.

All literature results identified in the search were uploaded into EndNote. Two reviewers (authors IHJE and $\mathrm{SJMvH}$ ) independently assessed abstracts to identify studies meeting the inclusion criteria for further review. In cases of disagreement, the study was included for full text review. All studies assessed as relevant were obtained in full text and reviewed independently by authors IHJE and SJMvH for definite inclusion according to the in- and exclusion criteria mentioned previously. In cases of disagreement, a third reviewer (author JCMvH) made the final decision on inclusion of studies based on the full text of the article.

\section{Data extraction and analysis}

Using a structured data-extraction form, one author (IHJE) extracted data from the included studies. The primary outcome measure was home discharge. Furthermore, extracted data were study design, sample characteristics (i.e., sample size, age and gender), primary diagnosis, 
rehabilitation setting, discharge destination, effect size of influencing factor and interpretation. The effect sizes of the influencing factors were considered significant if they had a p-value $\leq 0.05$. Data were categorized according to the factor that influenced home discharge.

In studies where multivariate statistical findings were presented, only these findings were extracted and incorporated into the data extraction table. In cases where only univariate statistical findings are included in the data extraction table this is an indication that the study did not display multivariate statistical findings.

\section{Methodological quality of identified studies}

Quality appraisal of the included studies was independently done by authors IHJE and JCMvH using the checklist for quality assessment of prognostic studies developed by Hayden and colleagues [20]. In cases of disagreement, results were discussed until consensus was reached. This checklist comprised six domains (A-F; see Additional file 2) and each of the six domains was subdivided into three to seven items. The exact meaning of these items can be retrieved in an additional file [see Additional file 2]. The items were scored with yes, partly, no, unsure or not applicable. 'Unsure' was used when the item was relevant for the type of study design but not clearly described by the authors. 'Not applicable' was used when the item was irrelevant for the study design and was therefore not possible to be described by the authors.

A domain scored two points if all items in the domain scored 'yes', or if one item was scored with 'partly' and the other items within the domain were scored with 'yes'. One point was allocated if the criteria necessary for receiving two points were not met but at least half of the items within the domain were scored with 'yes'. If more than half of the items of the domain were scored with 'partly', 'no', or 'unsure', the domain was allocated zero points. If at least $90 \%$ of the studies scored 'not applicable' on a specific item, that item was excluded from the domain.

Since there were six domains and a maximum of two points could be scored on each domain, the maximum possible score that could be gained was 12 . The authors of the present review considered a score of $75 \%$ (9 points) or higher to be a good methodological quality score. A score between $50-75 \%$ (6-8 points) was considered a moderate methodological quality score whereas a score below $50 \%$ (5 points or less) was considered a weak methodological quality score [21].

\section{Results}

\section{Included studies}

Figure 1 shows the flowchart of the study identification and selection process. After removing duplicates, 705 potentially relevant articles were identified. Subsequently, after screening for title and abstract, 666 articles were excluded because they did not meet the inclusion criteria. The full texts of the remaining 39 articles were assessed, which led to the exclusion of another 21 studies. Thus, in total 18 articles were included in the review.

\section{Methodological quality}

Table 1 shows the methodological quality of the 18 studies, based on the guidelines for assessing quality in prognostic studies by Hayden and colleagues [20]. The quality ranged from a score of 5 to 10 points (out of a theoretical range from 0 to 12), with a median of 7.5. After excluding the items that were not applicable in more than $90 \%$ of the studies, domain A, 'study participation', consisted of five items. Domain B, 'study attrition', had one item, domain $C$, 'prognostic factor measurement', had five items, domain D, 'outcome measurement', had three items, domain E, 'confounding measurement and account,' consisted of six items, and domain F, 'analysis', had three items.

Seven studies [22-28] had a score of at least $75 \%$ (9 points or more) of the total possible score of 12 . Another eight studies $[7,9,10,29-33]$ scored $50-75 \%(6-8$ points) of the maximum score of 12 , and three studies scored less than 6 points $[14,34,35]$, which the authors of the present study considered of weak methodological quality.

The full quality appraisal on all 30 items can be found in an additional file [see Additional file 3].

\section{Data extraction}

The characteristics of the studies are described in tables 2 and 3. Fifteen studies were conducted in the United States, one in Hong Kong, one in Israel and one in Australia. The sample sizes range from 119 to 63,793 participants. With one exception, all studies [31] included both male and female participants.

\section{Factors influencing home discharge after inpatient rehabilitation}

Twenty-four factors that potentially influenced discharge destination were identified (Table 3). Seven out of nine studies found a significant relationship between higher age and non-home discharge after inpatient rehabilitation $[10,23,28,29,32-34]$. The influence of ethnicity on home discharge was assessed in four studies. Three studies demonstrated that black and Hispanic ethnicity were significantly related to higher percentages of home discharge, compared to their white counterparts $[7,9,29]$ and one study did not report a significant relationship between ethnicity and home discharge [32]. Three studies investigated the association between marital status and discharge disposition. All of these studies revealed that being married is significantly related to home discharge $[9,29,31]$. Three studies indicated a positive association 


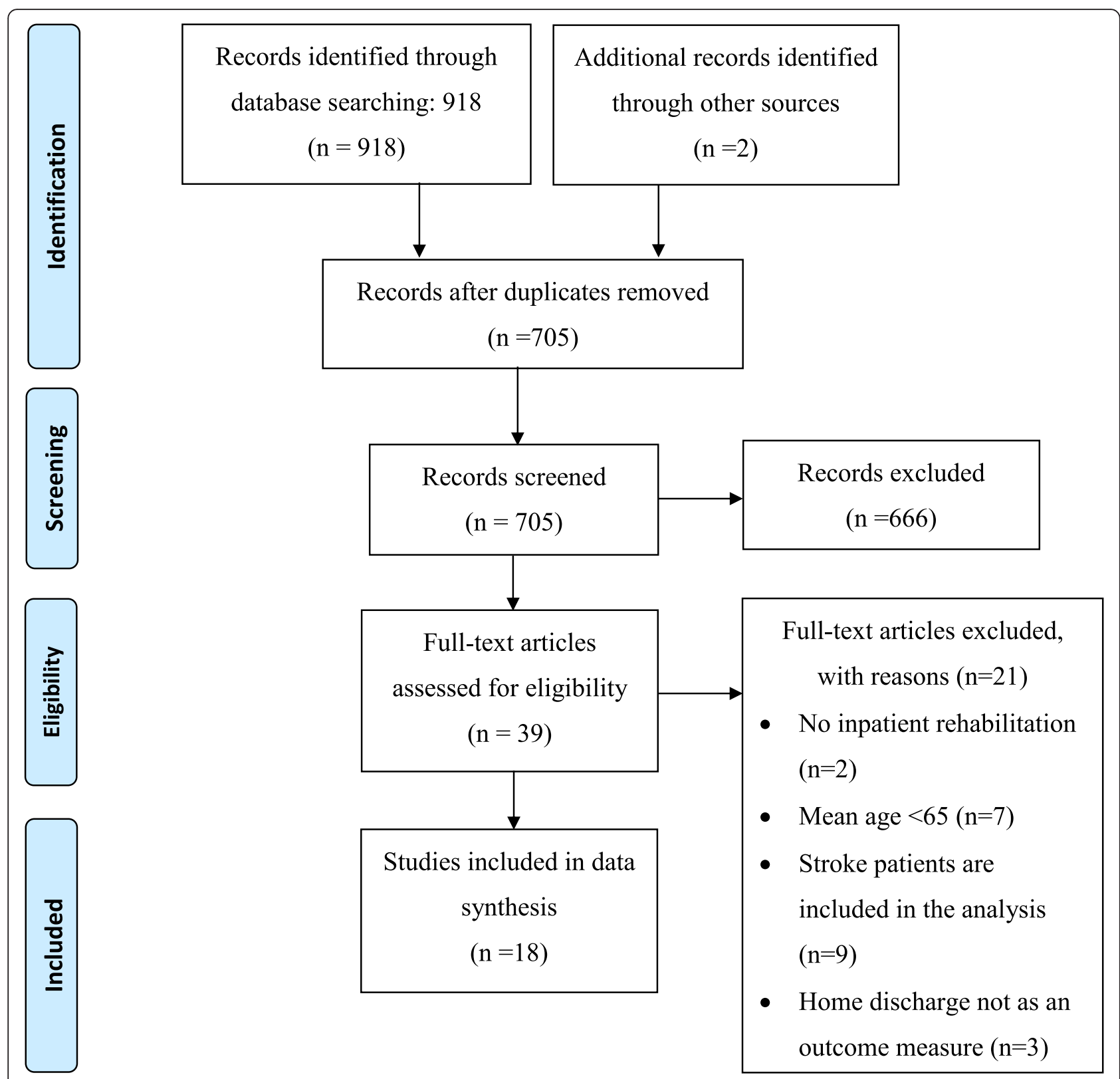

Fig. 1 Flowchart of the record identification and selection process

between higher functional status at admission and home discharge [10, 27, 28]. Furthermore, better cognitive function at admission was significantly related to home discharge in two out of three studies [30,32] and the presence of depression at admission was significantly related to discharge to a facility rather than home, which was shown by two studies [30, 31].

The relationship between living situation (alone or with someone else) and home discharge was assessed in two studies. One study [30] found a significant relationship between having a caregiver at home and home discharge, whereas the other study did not find such an association between living alone and home discharge compared with living with someone else [28] Four out of seven studies found a significant relationship between gender and home discharge after inpatient rehabilitation. Three studies reported a significant relationship between male gender and home discharge $[23,29,33]$, while one study revealed that being male is significantly related to non-home discharge [9].

Five out of six studies demonstrated the absence of a significant relationship between comorbidity and discharge destination [9, 10, 27-29] while one study claimed a negative significant relationship between congestive heart failure as a comorbid disease and home discharge [31]. An exception with respect to comorbidity is the influence of 
Table 1 Methodological quality assessment

\begin{tabular}{|c|c|c|c|c|c|c|c|c|}
\hline Author & Year & $A^{*}$ & $B^{* *}$ & $C$ & $D$ & $E$ & $\mathrm{~F}$ & Total(12) \\
\hline Berges [29] & 2008 & 1 & 2 & 1 & 1 & 1 & 1 & 7 \\
\hline Chang [9] & 2008 & 1 & 2 & 1 & 1 & 1 & 1 & 7 \\
\hline Chin [10] & 2008 & 2 & 2 & 1 & 1 & 1 & 1 & 8 \\
\hline Graham [7] & 2008 & 1 & 2 & 1 & 1 & 1 & 1 & 7 \\
\hline Hershkovitz [30] & 2007 & 1 & 2 & 2 & 1 & 1 & 1 & 8 \\
\hline Kay [22] & 2010 & 2 & 2 & 2 & 2 & 1 & 1 & 10 \\
\hline Kurichi [31] & 2010 & 1 & 2 & 1 & 1 & 1 & 1 & 7 \\
\hline New [23] & 2013 & 1 & 2 & 2 & 2 & 1 & 1 & 9 \\
\hline Sansone [27] & 2007 & 1 & 2 & 2 & 2 & 1 & 1 & 9 \\
\hline Siebens [32] & 2002 & 1 & 2 & 1 & 1 & 1 & 1 & 7 \\
\hline Vincent [33] & 2006 & 1 & 2 & 2 & 1 & 0 & 1 & 7 \\
\hline Vincent [25] & 2006 & 2 & 2 & 2 & 2 & 0 & 1 & 9 \\
\hline Vincent [26] & 2006 & 2 & 2 & 1 & 2 & 1 & 1 & 9 \\
\hline Vincent [24] & 2007 & 2 & 2 & 2 & 2 & 0 & 1 & 9 \\
\hline Vincent [34] & 2008 & 0 & 2 & 1 & 0 & 0 & 2 & 5 \\
\hline Vincent [35] & 2009 & 0 & 2 & 1 & 1 & 0 & 1 & 5 \\
\hline Vincent [14] & 2010 & 0 & 2 & 1 & 1 & 0 & 1 & 5 \\
\hline Yan [28] & 2013 & 1 & 2 & 2 & 2 & 1 & 1 & 9 \\
\hline
\end{tabular}

*Domain A, 'study participation', consists of five items, domain B, 'study attrition', has one item, domain $C$, 'prognostic factor measurement', has five items, domain $\mathrm{D}$, 'outcome measurement', has three items, domain $\mathrm{E}$, 'confounding measurement and account', has six items and domain F, 'analysis', has three items

**In domain B, four items were not used in the calculation because they were not applicable in more than $90 \%$ of the studies. In both domain $C$ and in domain $\mathrm{E}$, one item was not used in the calculation because it was not applicable in more than $90 \%$ of the items

obesity on home discharge, which was examined in four studies. None of the four studies demonstrated a significant relationship between obesity and discharge destination $[24,28,34,35]$.

\section{Discussion}

The findings from this systematic review show that home discharge after inpatient rehabilitation for geriatric patients is significantly related to younger age [10, 23, 28, 29, 32-34], non-white ethnicity $[7,9,29]$, being married $[9,29,31]$, higher functional $[10,27,28]$ and cognitive $[30,32]$ status and the absence of depression [30,31]. All predicting factors were measured at admission to the rehabilitation unit. Less clinical severity of the illness [32] and no active cardiac pathology [31] appeared to be significantly related to home discharge, however, these associations all come from only one study, therefore these results have to be treated with caution.

Due to inconsistent results, the association between home discharge and gender [9, 22, 23, 27-29, 33], comorbidity $[9,10,29,31]$, type of surgery $[10,25,26,34]$, living alone $[28,30]$ and postoperative complications $[10,31]$ was less obvious. These opposing outcomes might have been caused by differences in study populations (traumatic brain injury [9], hip replacement $[10,29,30,33]$, knee replacement [25, 28], spinal cord injury [23] and lower extremity amputation [31]) or a difference in the size of the study population [36]. Further research is required to explore the impact of these factors on home discharge after inpatient rehabilitation. In addition, no significant association was found between obesity and discharge disposition [24, 28, 34, 35]. The association between home discharge and the factors weight-bearing status at admission (restricted or not) [32], hematocrit value [14], travel distance from the inpatient rehabilitation facility [28], length of stay in the acute setting [10], pain [10], pre-fracture mobility status [10], the presence of a pressure sore [10], primary insurance [9], and smoking history [27] were also not significant. Because the evidence of these non-significant associations was based on single studies, further research into the impact of these factors is required. The three studies with weak methodological quality examined the association of higher age [34], type of surgery [34], Body Mass Index [34, 35] and hematocrit value [14] with home discharge. These effects might therefore also be treated with caution.

\section{Discriminative ability of methodological quality assessment domains}

The methodological quality of 15 out of 18 studies could be defined as moderate to good. However, the discriminative ability of four domains with respect to methodological quality is questionable. After excluding items that were 'not applicable' in at least $90 \%$ of the studies, domain B, 'study attrition', had only one item remaining. As a consequence, the score gained on that domain only ranged from 0 to 2. Since all included studies scored 2 points, this domain had no discriminative ability. The same holds for domain F focused on 'analysis'. Although this domain consisted of three items, all studies had a score of 1 , which again indicates a lack of discriminative ability. Furthermore, the scores on domain $\mathrm{C}$, 'prognostic factor measurement', and domain $\mathrm{E}$, 'confounding measurement and account', did not vary more than one point. It seems that, although assessing the methodological quality of the studies is done to differentiate between the quality of the included studies, some domains add very little to quality differences.

\section{Comparison with other research}

The findings from the present systematic review are in line with several prognostic factors for non-home discharge in stroke patients, as the review of Meijer and colleagues showed [15]. This latter review found that low initial activities of daily living (ADL) functioning, high age, cognitive disturbance, and being female predicted less 
Table 2 Characteristics of identified studies

\begin{tabular}{|c|c|c|c|c|c|}
\hline $\begin{array}{l}\text { Author, year, Country } \\
\text { [Reference Number] }\end{array}$ & Study design & $\begin{array}{l}\text { Sample characteristics } \\
\text { ( } n \text {, age, gender) }\end{array}$ & Primary diagnosis & Rehabilitation setting & Discharge destination \\
\hline $\begin{array}{l}\text { Bergés et al., 2008, } \\
\text { United States [29] }\end{array}$ & Retrospective cohort study & $\begin{array}{l}n=63,793 ; \text { mean age }=71.7 ; \\
66.5 \% \text { female }\end{array}$ & Hip replacement & Inpatient rehabilitation facilities & Home vs. not home \\
\hline $\begin{array}{l}\text { Chang et al., 2008, } \\
\text { United States [9] }\end{array}$ & Retrospective cohort study & $\begin{array}{l}N=9,240^{\mathrm{a}} ; \text { mean age }=78.8 ; \\
47 \% \text { female }\end{array}$ & Traumatic brain injury & Inpatient rehabilitation facilities & Home vs. not home \\
\hline $\begin{array}{l}\text { Chin et al., 2008; } \\
\text { Hong Kong [10] }\end{array}$ & Prospective cohort study & $\begin{array}{l}n=303, \text { mean age }=82, \\
70 \% \text { female }\end{array}$ & Hip fracture & Inpatient rehabilitation hospital & Home vs. not home \\
\hline $\begin{array}{l}\text { Graham et al., 2008; } \\
\text { United States [7] }\end{array}$ & Retrospective cohort study & $\begin{array}{l}N=42,479 ; \text { mean age }=82.2 ; \\
31.4 \% \text { female }\end{array}$ & Hip fracture & Inpatient rehabilitation facilities & Home vs. not home \\
\hline $\begin{array}{l}\text { Hershkovitz et al.,2007; } \\
\text { Israel [30] }\end{array}$ & Prospective cohort study & $\begin{array}{l}N=133 ; \text { mean age }=80 ; \\
79.7 \% \text { female }\end{array}$ & Hip fracture & $\begin{array}{l}\text { Rehabilitation unit of a geriatric } \\
\text { hospital }\end{array}$ & Home vs. nursing home \\
\hline $\begin{array}{l}\text { Kay et al., 2010; } \\
\text { United States [22] }\end{array}$ & Retrospective cohort study & $\begin{array}{l}N=1,645, \text { mean age }=70 ; \\
57.1 \% \text { female }\end{array}$ & Non-traumatic spinal cord injury & Inpatient rehabilitation facility & Residence vs. nursing home \\
\hline $\begin{array}{l}\text { Kurichi et al., 2013; } \\
\text { United States [31] }\end{array}$ & Retrospective observational study & $\begin{array}{l}N=1,480^{\mathrm{b}}, \text { mean age }=66.8 ; \\
100 \% \text { male }\end{array}$ & Lower extremity amputation & Veterans Affairs Medical Centers & Home vs. not home \\
\hline $\begin{array}{l}\text { New, 2007; } \\
\text { Australia [23] }\end{array}$ & Retrospective, 3-year case series & $\begin{array}{l}N=70, \text { mean age }=65 \\
54.3 \% \text { female }\end{array}$ & Non-traumatic spinal cord injury & Tertiary medical unit & Home vs. not home \\
\hline $\begin{array}{l}\text { Sansone et al., 2002; } \\
\text { United States [27] }\end{array}$ & Retrospective study & $\begin{array}{l}N=143, \text { median age }=70 \\
39.9 \% \text { female }\end{array}$ & Cardiac patients & Public acute long-term care hospital & Home vs. not home \\
\hline $\begin{array}{l}\text { Siebens et al., 2012, } \\
\text { United States [32] }\end{array}$ & $\begin{array}{l}\text { Multi-site prospective observational } \\
\text { cohort study }\end{array}$ & $\begin{array}{l}N=224 ; \text { mean age }=76.8 \\
78 \% \text { female }\end{array}$ & Hip fracture & $\begin{array}{l}9 \text { skilled nursing facilities and } 11 \\
\text { inpatient rehabilitation facilities }\end{array}$ & Home vs. not home \\
\hline $\begin{array}{l}\text { Vincent et al., 2006; } \\
\text { United States [33] }\end{array}$ & Retrospective study & $\begin{array}{l}N=332, \text { mean age }=70.6 \\
63.6 \% \text { female }\end{array}$ & Total hip arthroplasty & Inpatient rehabilitation hospital & Home vs. not home \\
\hline $\begin{array}{l}\text { Vincent et al., 2006; } \\
\text { United States [25] }\end{array}$ & Retrospective study & $\begin{array}{l}N=424 ; \text { mean age }=70.7 ; \\
70 \% \text { female }\end{array}$ & Total knee arthroplasty & Inpatient rehabilitation hospital & Home vs. not home \\
\hline $\begin{array}{l}\text { Vincent et al., 2006; } \\
\text { United states [26] }\end{array}$ & Retrospective study & $\begin{array}{l}N=402 ; \text { mean age }=70.8 \\
62.9 \% \text { female }\end{array}$ & Total hip arthroplasty & Inpatient rehabilitation hospital & Home vs. not home \\
\hline $\begin{array}{l}\text { Vincent et al., 2007; } \\
\text { United States [24] }\end{array}$ & Retrospective, comparative study & $\begin{array}{l}N=146 ; \text { mean age }=70.8 \\
70.1 \% \text { female }\end{array}$ & Total knee arthroplasty & Inpatient rehabilitation hospital & Home vs. not home \\
\hline $\begin{array}{l}\text { Vincent et al., 2008; } \\
\text { United States [34] }\end{array}$ & Retrospective study & $\begin{array}{l}N=23,649, \text { mean age }=70.2 ; \\
\text { Female }=67 \%\end{array}$ & Total hip or knee arthroplasty & Inpatient rehabilitation facility & Home vs. not home \\
\hline $\begin{array}{l}\text { Vincent et al., 2009; } \\
\text { United States [35] }\end{array}$ & Retrospective, comparative study & $\begin{array}{l}N=1,947, \text { mean age }=71 \\
\text { female }=70.5 \%\end{array}$ & Total hip arthroplasty & Inpatient rehabilitation facilities & Home vs. not home \\
\hline $\begin{array}{l}\text { Vincent et al., 2010; } \\
\text { United States [14] }\end{array}$ & Multicenter, retrospective study & $\begin{array}{l}N=5,421, \text { mean age }=69.8 \\
68.6 \% \text { female }\end{array}$ & Total knee arthroplasty & 15 Inpatient rehabilitation facilities & Home vs. not home \\
\hline $\begin{array}{l}\text { Yan et al., 2013; } \\
\text { United States [28] }\end{array}$ & Retrospective study & $\begin{array}{l}N=119 ; \text { mean age }=67.4 ; \\
5.9 \% \text { female }\end{array}$ & $\begin{array}{l}\text { Total knee arthroplasty/bilateral knee } \\
\text { surgery/total hip arthroplasty }\end{array}$ & $\begin{array}{l}\text { Inpatient rehabilitation in a Veterans } \\
\text { Affairs Medical Center }\end{array}$ & Home vs. not home \\
\hline
\end{tabular}

vs. stands for 'versus'. "Home" discharge means discharge to home, the community or an assisted living facility. Discharge to "not home" means discharge to a variety of inpatient care facilities, such as a skilled nursing facility, a nursing home, or acute care

a Before hospitalization, $8 \%$ of the total population came from intermediate care or another hospital

${ }^{\mathrm{b}}$ Before hospitalization, $1.5 \%$ of the total population lived in an institution 
Table 3 Data extraction of identified studies

\begin{tabular}{|c|c|c|c|c|c|}
\hline Influencing factor & Study & Influencing factor specified & Discharge destination & Result & Interpretation \\
\hline \multirow[t]{9}{*}{ Age } & Bergés et al., 2008 [29] & Higher age & Home vs. institution & $\mathrm{OR}=0.97(0.97-0.98)^{*}$ & $\begin{array}{l}\text { Higher age is related to fewer } \\
\text { home discharges }\end{array}$ \\
\hline & Chang et al., 2008 [9] & Each 1 year increase in age & Home vs. institution & $\mathrm{OR}=0.99(0.99-1.00)$ & $\begin{array}{l}\text { Higher age is not related to } \\
\text { discharge disposition }\end{array}$ \\
\hline & Chin et al., 2008 [10] & Age $\geq 80$ years & Institution vs. home & $\mathrm{OR}=1.92(1.04-3.57)^{*}$ & $\begin{array}{l}\text { Higher age is related to } \\
\text { discharge to an institution }\end{array}$ \\
\hline & New, 2007 [23] & Higher age & Home vs. institution & Wilkinson-rank sum test; $p=0.01^{*}$ & $\begin{array}{l}\text { Higher age is related to fewer } \\
\text { home discharges }\end{array}$ \\
\hline & Sansone et al., 2002 [27] & Age & Home vs. institution & $\mathrm{OR}=1.05(0.99-1.09)$ & $\begin{array}{l}\text { Age is not related to discharge } \\
\text { disposition }\end{array}$ \\
\hline & Siebens et al., 2012 [32] & Higher age & Home vs. institution & $\mathrm{OR}=0.92(0.87-0.96)^{*}$ & $\begin{array}{l}\text { Higher age is related to fewer } \\
\text { home discharges }\end{array}$ \\
\hline & Vincent et al., 2006 [33] & Age group $<85$ vs. $\geq 85$ & Home vs. institution & $x^{2} ; p<0.05^{*}$ & $\begin{array}{l}\text { Higher age is related to fewer } \\
\text { home discharges }\end{array}$ \\
\hline & Vincent et al., 2008 [34] & Age group $<85$ vs. $\geq 85$ & Non-home vs. home & $\mathrm{OR}=3.63(3.37-3.89) \neq$ & $\begin{array}{l}\text { Higher age is related to fewer } \\
\text { home discharges }\end{array}$ \\
\hline & Yan et al., 2013 [28] & Younger age & Home vs. not home & MANOVA; $p=0.04^{*}$ & $\begin{array}{l}\text { Younger age is related to } \\
\text { home discharge }\end{array}$ \\
\hline \multirow[t]{9}{*}{ Ethnicity } & Bergés et al., 2008 [29] & Black vs. white & Home vs. institution & $\mathrm{OR}=1.23(1.07-1.41)^{*}$ & $\begin{array}{l}\text { Black race is related to home } \\
\text { discharge }\end{array}$ \\
\hline & & Hispanic vs. white & & $\mathrm{OR}=1.51(1.15-1.99)^{*}$ & $\begin{array}{l}\text { Hispanic race is related to } \\
\text { home discharge }\end{array}$ \\
\hline & & Asian vs. white & & $\mathrm{OR}=1.67(0.93-3.00)$ & $\begin{array}{l}\text { Asian race is not related to } \\
\text { discharge disposition }\end{array}$ \\
\hline & Chang et al., 2008 [9] & Black vs. white & Home vs. institution & $\mathrm{OR}=2.00(1.55-2.59)^{*}$ & $\begin{array}{l}\text { Black race is related to home } \\
\text { discharge }\end{array}$ \\
\hline & & Hispanic vs. white & & $\mathrm{OR}=2.24(1.66-3.02)^{*}$ & $\begin{array}{l}\text { Hispanic race is related to } \\
\text { home discharge }\end{array}$ \\
\hline & Graham et al., 2008 [7] & Non-Hispanic black vs. white & Home vs. institution & $\mathrm{OR}=2.02(1.77-2.32)^{*}$ & $\begin{array}{l}\text { Non-Hispanic Black race is } \\
\text { related to home discharge }\end{array}$ \\
\hline & & Hispanic vs. white & & $\mathrm{OR}=1.90(1.64-2.19)^{*}$ & $\begin{array}{l}\text { Hispanic race is related to } \\
\text { home discharge }\end{array}$ \\
\hline & & Asian vs. white & & $\mathrm{OR}=2.07(1.55-2.78)^{*}$ & $\begin{array}{l}\text { Asian race is related to home } \\
\text { discharge }\end{array}$ \\
\hline & Siebens et al., 2012 [32] & Nonwhite vs. white & Home vs. institution & $\mathrm{OR}=4.34(0.86-21.79)$ & $\begin{array}{l}\text { Nonwhite race is not related } \\
\text { discharge disposition }\end{array}$ \\
\hline \multirow[t]{2}{*}{ Marital status } & Bergés et al., 2008 [29] & Married vs. not-married & Home vs. institution & $\mathrm{OR}=2.42(2.24-2.61)^{*}$ & $\begin{array}{l}\text { Being married is related to } \\
\text { home discharge }\end{array}$ \\
\hline & Chang et al., 2008 [9] & Not-married vs. married & Home vs. institution & $\mathrm{OR}=0.45(0.40-0.51)^{*}$ & $\begin{array}{l}\text { Being not-married is related to } \\
\text { fewer home discharges }\end{array}$ \\
\hline
\end{tabular}


Table 3 Data extraction of identified studies (Continued)

\begin{tabular}{|c|c|c|c|c|c|}
\hline & Kurichi et al., 2013 [30] & Married vs. not-married & Home vs. institution & $\mathrm{OR}=1.51(1.14-1.99)^{* *}$ & $\begin{array}{l}\text { Being married is related to } \\
\text { home discharge }\end{array}$ \\
\hline \multirow[t]{3}{*}{ Functional status } & Chin et al., 2008 [10] & Admission FIM score $<75$ & Institution vs. home & $\mathrm{OR}=4.68(2.23-9.82)^{*}$ & $\begin{array}{l}\text { Lower functional status at } \\
\text { admission is related to } \\
\text { discharge to an institution }\end{array}$ \\
\hline & Sansone et al., 2002 [27] & $\begin{array}{l}\text { Admission FIM score } \leq 71 \mathrm{vs} \\
\text { admission FIM score }>72\end{array}$ & Home vs. institution & $\mathrm{OR}=0.91(0.85-0.96)^{* * *}$ & $\begin{array}{l}\text { Lower functional status at } \\
\text { admission is related to fewer } \\
\text { home discharges }\end{array}$ \\
\hline & Yan et al., 2013 [28] & Admission FIM score & Home vs. institution & MANOVA $p=0.00^{* * *}$ & $\begin{array}{l}\text { Higher functional status at } \\
\text { admission is related to home } \\
\text { discharge }\end{array}$ \\
\hline \multirow[t]{3}{*}{ Cognition } & Chin et al., 2008 [10] & Admission AMT <6 & Institution vs. home & $\mathrm{OR}=1.60(0.87-2.96)$ & $\begin{array}{l}\text { Impaired cognitive function at } \\
\text { admission is not related to } \\
\text { discharge disposition }\end{array}$ \\
\hline & Hershkovitz et al., 2007 [30] & MMSE score & Home vs. nursing home & $\mathrm{OR}=1.11(1.03-1.20)^{*}$ & $\begin{array}{l}\text { Higher cognitive functional } \\
\text { level is related to home } \\
\text { discharge }\end{array}$ \\
\hline & Siebens et al., 2012 [32] & Cognitive FIM at admission & Home vs. institution & $\mathrm{OR}=1.06(1.01-1.11)^{*}$ & $\begin{array}{l}\text { Better cognitive function at } \\
\text { admission is related to home } \\
\text { discharge }\end{array}$ \\
\hline \multirow[t]{2}{*}{ Depression } & Hershkovitz et al., 2007 [30] & Presence of depression & Home vs. nursing home & $\mathrm{OR}=0.30(0.11-0.84)^{*}$ & $\begin{array}{l}\text { The presence of depression is } \\
\text { related to fewer home } \\
\text { discharges }\end{array}$ \\
\hline & Kurichi et al., 2013 [31] & Presence of depression & Home vs. institution & $\mathrm{OR}=0.63(0.40-0.98)^{*}$ & $\begin{array}{l}\text { The presence of depression is } \\
\text { related to fewer home } \\
\text { discharges }\end{array}$ \\
\hline Clinical severity of illness & Siebens et al., 2012 [32] & Lower maximum severity & Home vs. institution & $\mathrm{OR}=0.95(0.93-0.97)^{* * *}$ & $\begin{array}{l}\text { Lower severity of illness } \\
\text { calculated by the CSI is related } \\
\text { to home discharge }\end{array}$ \\
\hline Treatment-level procedures & Kurichi et al., 2013 [31] & $\begin{array}{l}\text { Ongoing active cardiac pathology } \\
\text { (yes vs. no) }\end{array}$ & Home vs. institution & $\mathrm{OR}=0.55(0.37-0.81)^{* *}$ & $\begin{array}{l}\text { Treatment level procedure is } \\
\text { related to home discharge }\end{array}$ \\
\hline \multirow[t]{2}{*}{ Pre-hospital living situation } & Yan et al., 2013 [28] & Lives alone vs. lives with someone & Home vs. institution & Fisher's Exact Text: $p=0.35$ & $\begin{array}{l}\text { Living alone is not related to } \\
\text { discharge disposition }\end{array}$ \\
\hline & Hershkovitz et al., 2007 [30] & Presence of a caregiver at home & Home vs. nursing home & $\mathrm{OR}=8.88(1.76-44.9)^{*}$ & $\begin{array}{l}\text { The presence of a caregiver at } \\
\text { home is related to home } \\
\text { discharge }\end{array}$ \\
\hline \multirow[t]{3}{*}{ Gender } & Bergés et al., 2008 [29] & Male vs. female & Home vs. institution & $\mathrm{OR}=1.08(1.01-1.17)^{*}$ & $\begin{array}{l}\text { Male gender is related to } \\
\text { home discharge }\end{array}$ \\
\hline & Chang et al., 2008 [9] & Male vs. female & Home vs. institution & $\mathrm{OR}=0.85(0.75-0.96)^{*}$ & $\begin{array}{l}\text { Male gender is related to fewer } \\
\text { home discharges }\end{array}$ \\
\hline & Kay et al., 2010 [22] & $\begin{array}{l}\text { Male vs. female; etiology } \\
\text { degenerative spinal disorders }\end{array}$ & $\begin{array}{l}\text { Community-based residence } \\
\text { vs. nursing home }\end{array}$ & $\mathrm{OR}=1.00(0.50-1.99)$ & $\begin{array}{l}\text { Gender is not related to } \\
\text { discharge disposition }\end{array}$ \\
\hline
\end{tabular}


Table 3 Data extraction of identified studies (Continued)

\begin{tabular}{|c|c|c|c|c|c|}
\hline & & & & $\mathrm{OR}=0.98(0.39-2.45)$ & $\begin{array}{l}\text { Gender is not related to } \\
\text { discharge disposition }\end{array}$ \\
\hline & & \multicolumn{2}{|l|}{$\begin{array}{l}\text { Male vs. female; etiology malignant } \\
\text { tumor }\end{array}$} & $\mathrm{OR}=0.73(0.22-2.49)$ & $\begin{array}{l}\text { Gender is not related to } \\
\text { discharge disposition }\end{array}$ \\
\hline & & \multicolumn{2}{|l|}{$\begin{array}{l}\text { Male vs. female; etiology vascular } \\
\text { ischemia }\end{array}$} & & \\
\hline & New, 2007 [23] & Male vs. female & Home vs. not home & $x^{2} ; p=0.00^{* *}$ & $\begin{array}{l}\text { Female gender is related to } \\
\text { fewer home discharges }\end{array}$ \\
\hline & Sansone et al., 2002 [27] & Male vs. female & Home vs. institution & $\mathrm{OR}=1.01(0.35-2.95)$ & $\begin{array}{l}\text { Gender is not related to } \\
\text { discharge disposition }\end{array}$ \\
\hline & Vincent et al., 2006 [33] & Female vs. male & Home vs. institution & $x^{2} ; p<0.05^{*}$ & $\begin{array}{l}\text { Female gender is related to } \\
\text { fewer home discharges }\end{array}$ \\
\hline & Yan et al., 2013 [28] & Male vs. female & Home vs. institution & Fisher's Exact Test; $p=0.27$ & $\begin{array}{l}\text { Male gender is not related to } \\
\text { discharge disposition }\end{array}$ \\
\hline \multirow[t]{7}{*}{ Comorbidity } & Berges et al., 2008 [29] & One or more & Home vs. institution & $\mathrm{OR}=1.14(0.83-1.57)$ & $\begin{array}{l}\text { The presence of one or more } \\
\text { comorbidities is not related to } \\
\text { discharge disposition }\end{array}$ \\
\hline & \multirow[t]{2}{*}{ Chang et al., 2008 [9] } & \multirow[t]{2}{*}{$1-3>3$} & \multirow[t]{2}{*}{ Home vs. institution } & $\mathrm{OR}=1.09(0.73-1.63)$ & \multirow{2}{*}{$\begin{array}{l}\text { The presence of one or more } \\
\text { comorbidities is not related to } \\
\text { discharge disposition }\end{array}$} \\
\hline & & & & $\mathrm{OR}=1.35(0.95-1.93)$ & \\
\hline & Chin et al., 2008 [10] & CVA or Parkinsonism & Institution vs. home & $\mathrm{OR}=1.18(0.56-2.51)$ & $\begin{array}{l}\text { The presence of CVA or } \\
\text { Parkinsonism as a comorbidity } \\
\text { is not related to discharge } \\
\text { disposition }\end{array}$ \\
\hline & Kurichi et al., 2013 [31] & Congestive heart failure & Home vs. institution & $\mathrm{OR}=0.62(0.45-0.85)^{* *}$ & $\begin{array}{l}\text { The presence of congestive } \\
\text { heart failure as a comorbidity } \\
\text { is associated with fewer home } \\
\text { discharges }\end{array}$ \\
\hline & Sansone et al., 2002 [27] & 1 or more vs. 0 & Home vs. institution & $\mathrm{OR}=1.13(0.37-3.38)$ & $\begin{array}{l}\text { The presence of a comorbidity } \\
\text { is not associated with } \\
\text { discharge disposition }\end{array}$ \\
\hline & Yan et al., 2013 [28] & Number of comorbidities & Home vs. institution & MANOVA $p=0.32$ & $\begin{array}{l}\text { The number of comorbidities } \\
\text { is not associated with } \\
\text { discharge disposition }\end{array}$ \\
\hline \multirow[t]{3}{*}{ Type of surgery } & Chin et al., 2008 [10] & $\begin{array}{l}\text { Arthroplasty vs. Closed Reduction } \\
\text { Internal Fixation (CRIF) }\end{array}$ & Institution vs. home & $\mathrm{OR}=0.99(0.56-1.73)$ & $\begin{array}{l}\text { Fracture management is not } \\
\text { related to discharge disposition }\end{array}$ \\
\hline & Vincent et al., 2006 [26] & $\begin{array}{l}\text { Home discharge in the primary } \\
\text { total hip arthroplasty (THA) } \\
\text { group vs. the revision THA group }\end{array}$ & Home vs. institution & Kruskal-Wallis; $p<0.00^{* * *}$ & $\begin{array}{l}\text { Type of surgery in hip } \\
\text { arthroplasty patients is related } \\
\text { to home discharge }\end{array}$ \\
\hline & Vincent et al., 2006 [25] & $\begin{array}{l}\text { Home discharge in the primary } \\
\text { total knee arthroplasty (TKA) } \\
\text { group vs. the revision TKA group }\end{array}$ & Home vs. institution & Kruskal-Wallis; $p<0.00^{* * *}$ & $\begin{array}{l}\text { Type of surgery in knee } \\
\text { arthroplasty patients is related } \\
\text { to home discharge }\end{array}$ \\
\hline
\end{tabular}


Table 3 Data extraction of identified studies (Continued)

\begin{tabular}{|c|c|c|c|c|c|}
\hline & Vincent et al., 2008 [34] & $\begin{array}{l}\text { Bilateral joint procedures (THA + } \\
\text { TKA) or unilateral joint procedures }\end{array}$ & Home vs. institution & $\mathrm{OR}=0.76(0.49-1.01)$ & $\begin{array}{l}\text { The type of joint procedure is } \\
\text { not related to discharge } \\
\text { disposition }\end{array}$ \\
\hline \multirow[t]{2}{*}{ Postoperative complications } & Chin et al., 2008 [10] & $\begin{array}{l}\text { Chest infection or urinary tract } \\
\text { infection }\end{array}$ & Institution vs. home & $\mathrm{OR}=1.44(0.56-3.69)$ & $\begin{array}{l}\text { The postoperative complications } \\
\text { chest infection or urinary tract } \\
\text { infection are not related to } \\
\text { discharge disposition }\end{array}$ \\
\hline & Kurichi et al., 2013 [31] & $\begin{array}{l}\text { Local significant infection at } \\
\text { amputation }\end{array}$ & Home vs. institution & $\mathrm{OR}=0.57(0.39-0.83)^{* *}$ & $\begin{array}{l}\text { Postoperative complications } \\
\text { are related to fewer home } \\
\text { discharges }\end{array}$ \\
\hline Admission weight-bearing status & Siebens et al., 2012 [32] & $\begin{array}{l}\text { Weight bearing as tolerated } \\
\text { (WBAT) vs. restricted weight } \\
\text { bearing (RWB) after hip fracture }\end{array}$ & Home vs. institution & $\mathrm{OR}=2.58(0.99-6.70)$ & $\begin{array}{l}\text { Admission status "weight } \\
\text { bearing as tolerated" is not } \\
\text { related to discharge disposition }\end{array}$ \\
\hline Hematocrit value & Vincent et al., 2010 [14] & $\begin{array}{l}\text { Very low hematocrit } \\
\text { (Hct }<30 \% \text { ) vs. low Hct } \\
\text { (30-36\% women; } 30-41 \% \\
\text { men) vs. normal Hct } \\
\text { (>36\% women; >41 \% men) }\end{array}$ & Home vs. institution & $x^{2} ; p>0.05$ & $\begin{array}{l}\text { Hematocrit value is not related } \\
\text { to discharge disposition }\end{array}$ \\
\hline Distance & Yan et al., 2013 [28] & $\begin{array}{l}\text { Distance from inpatient } \\
\text { rehabilitation facility in miles }\end{array}$ & Home vs. institution & MANOVA $p=0.09$ & $\begin{array}{l}\text { The distance from the inpatient } \\
\text { rehabilitation facility is not } \\
\text { related to discharge disposition }\end{array}$ \\
\hline Length of Stay in acute setting & Chin et al., 2008 [10] & $>7$ days & Institution vs. home & $\mathrm{OR}=1.05(0.59-1.87)$ & $\begin{array}{l}\text { The length of stay in the acute } \\
\text { setting is not related to } \\
\text { discharge disposition }\end{array}$ \\
\hline \multirow[t]{4}{*}{ Obesity } & Vincent et al., 2007 [24] & $\mathrm{BMl}<30 \mathrm{~kg} / \mathrm{m}^{2}$ vs. BMl $\geq 30 \mathrm{~kg} / \mathrm{m}^{2}$ & Home vs. institution & $x^{2} ; p>0.05$ & $\begin{array}{l}\text { Obesity is not related to } \\
\text { discharge disposition }\end{array}$ \\
\hline & Vincent et al., 2008 [34] & $\mathrm{BMI} \geq 50 \mathrm{~kg} / \mathrm{m}^{2}$ vs. $\mathrm{BMI}<50 \mathrm{~kg} / \mathrm{m}^{2}$ & Home vs. institution & $\mathrm{OR}=0.97(0.71-1.23)$ & $\begin{array}{l}\text { BMl is not related to discharge } \\
\text { disposition }\end{array}$ \\
\hline & Vincent et al., 2009 [35] & $\begin{array}{l}\mathrm{BMl}<25 \mathrm{~kg} / \mathrm{m}^{2} \text { vs. BMI } 25-29.9 \mathrm{~kg} / \mathrm{m}^{2} \\
\text { vs BMl } 30-40 \mathrm{~kg} / \mathrm{m}^{2} \text { vs. BMl }>40 \mathrm{~kg} / \mathrm{m}^{2}\end{array}$ & Home vs. institution & $x^{2} ; p>0.05$ & $\begin{array}{l}\text { BMI is not related to discharge } \\
\text { disposition }\end{array}$ \\
\hline & Yan et al., 2013 [28] & $\begin{array}{l}\text { Difference in BMI between home } \\
\text { discharge and not home discharge }\end{array}$ & Home vs. institution & MANOVA $p=0.78$ & $\begin{array}{l}\text { BMI is not related to discharge } \\
\text { disposition }\end{array}$ \\
\hline Pain & Chin et al., 2008 [10] & VAS pain scale at admission $\geq 4$ & Institution vs. home & $\mathrm{OR}=0.61(0.33-1.13)$ & $\begin{array}{l}\text { Higher pain score at admission } \\
\text { is not related to discharge } \\
\text { disposition }\end{array}$ \\
\hline Pre-fracture mobility status & Chin et al., 2008 [10] & Dependent or non-walker & Institution vs. home & $\mathrm{OR}=1.84(0.94-3.60)$ & $\begin{array}{l}\text { Pre-fracture dependent mobility } \\
\text { status is not related to discharge } \\
\text { disposition }\end{array}$ \\
\hline Pressure sore & Chin et al., 2008 [10] & $\begin{array}{l}\text { Pressure sore at admission to } \\
\text { rehabilitation }\end{array}$ & Institution vs. home & $\mathrm{OR}=1.10(0.44-2.73)$ & $\begin{array}{l}\text { The presence of a pressure sore } \\
\text { at admission is not related to } \\
\text { discharge disposition }\end{array}$ \\
\hline Primary insurance & Chang et al., 2008 [9] & Private vs. Medicare & Home vs. institution & $\mathrm{OR}=1.01(0.81-1.25)$ & \\
\hline
\end{tabular}


Table 3 Data extraction of identified studies (Continued)

Medicaid vs. Medicare

$\mathrm{OR}=1.01(0.45-2.28)$

$\mathrm{OR}=1.23(0.70-2.17)$

The type of primary insurance

Other vs. Medicare

disposition

Smoking history

Sansone et al., 2002 [27]

Smoker vs. non-smoker

Home vs. institution

$\mathrm{OR}=3.17(0.86-11.63)$

Smoking history is not related to discharge disposition

vs. stands for versus; CVA denotes cerebrovascular accident; FIM Functional independence measure; AMT Abbreviated Mental Test; MMSE Mini Mental State Examination; CSI Comprehensive Severity Index and VAS Visual Analogue Scale

${ }^{*} P<0.05$

${ }^{* *} p<0.01$

${ }^{* * *} p<0.001$ 
home discharge in the sub-acute phase after stroke [15]. Other factors associated with home discharge were strokerelated factors such as paresis of arm and leg, initial level of consciousness being 'not alert' and constructional apraxia; therefore, these results cannot be compared with the results of the present review.

Factors affecting discharge destination in older medical patients who return home after hospital admission without inpatient rehabilitation are also comparable as presented in a systematic literature review by Campbell and colleagues [37]. Their review showed significant findings for functional status, cognitive functioning and age in relation to discharge destination. Gender and comorbidity appeared to have no significant relationship with discharge destination [37].

Although this review revealed that ethnicity seems to have a significant influence on home discharge, ethnicity is not addressed in the reviews from Meijer and colleagues [15] and Campbell and colleagues [37].

\section{Issues to be considered}

Some issues in this study need to be considered. First, we included studies with various patient populations. Although this is a good reflection of the heterogeneous population in rehabilitation, it is a methodological challenge because this hampers the comparability of the studies, and it is not clear whether a relationship observed in a specific diagnosis group will also be present in another diagnosis group. For this reason, we performed a subgroup analysis among the 13 studies that included only patients with orthopedic disorders. When analyzing the factors influencing home discharge among this subgroup, younger age, non-white ethnicity, higher functional and cognitive status still appear to be of significant value (the results are supported by at least two studies). The statistical significant effects of marital status and the absence of depression on home discharge are both supported by only one study in this subgroup analysis, and should therefore be treated with caution. This implies that, although minor differences exist, the factors influencing home discharge among the different diagnosis groups seem to be fairly comparable and may therefore be interpreted as rather robust. Apart from ethnicity, these results are also in line with influencing factors of home discharge among the stroke population [15].

Overall, our review found 23 possible influencing factors of home discharge after inpatient rehabilitation for geriatric patients but only six factors demonstrated a clear significant and rather consistent association. Therefore, future research into the inconsistent factors and into the factors that were only examined by one study is warranted.

\section{Study limitations}

First, the quantity, intensity and quality of therapies offered within inpatient rehabilitation for older patients might differ between countries and between rehabilitation units, the received therapy was not described in the included studies and could therefore not be taken into account in this review. Despite the differences in the included studies in diagnosis, received therapy and admission rules, several predicting factors were rather similar across patients and settings thus showing their robustness as well.

Second, the validity of systematic reviews is dependent on the absence of publication bias [38]. The presentation of only those results that are significant with non-significant results being excluded from publication, could lead to misleading conclusions. Therefore, the risk of publication bias should always be taken into account when results are interpreted. Third, there is always a risk of missing studies because they were not identified by the search strategy. We tried to minimize this potential bias by not only screening articles identified by the databases, but by analyzing reference lists of included articles as well.

Another limitation of our study is that the data extraction has been conducted by one researcher instead of two researchers independently, which could affect rigor. Furthermore, analytic strategies in the included studies varied; both multivariate and univariate outcomes are presented. Although this is accounted for in the methodological quality assessment, it means that some studies adjusted for confounders while others did not.

Finally, the protocol of our study has not been registered or published. Because the methods used did not change during the course of the study, we believe that this did not affect our results.

\section{Conclusions}

To help care professionals in setting more realistic rehabilitation goals and in preparing patients and informal caregivers for probable changes in living arrangement after discharge, we recommend assessing at least the following factors during admission of older patients to a rehabilitation unit: age, marital status, presence of depression, level of cognitive functioning and functional status. This assessment will help care professionals to make a more reliable prediction of discharge destination and to optimally tailor the rehabilitation treatment to the needs of the patient and their family. Because the prognostic factors of home discharge among stroke patients appear to be comparable to those of non-stroke patients, this assessment can be applied to all older patients admitted to an inpatient rehabilitation unit.

\section{Additional files}

Additional file 1: Search strategy. (DOCX $15 \mathrm{~kb})$ 
Additional file 2: Methodological quality assessment items. (DOCX $18 \mathrm{~kb}$ )

\section{Additional file 3: Methodological quality assessment of included} studies. (DOCX $28 \mathrm{~kb}$ )

\section{Competing interests}

The authors declare that they have no competing interests.

\section{Authors' contributions}

IHJE is the guarantor of the review. All authors take responsibility for the truthfulness of the data and the precision of the data analysis, the outline and critical revision of the manuscript. Contributions: IHJE participated in the literature search and inclusion of studies, the assessment of methodological quality, data analysis and writing the manuscript. JCMvH participated in the assessment of methodological quality and writing the manuscript. SJMvH participated in the literature search and the inclusion of studies. JMGAS participated in writing the manuscript. GIJMK participated in writing the manuscript. All authors contributed to the study concept and design and critical revision of the manuscript. All authors read and approved the final manuscript and agree to be accountable for all aspects of the work.

\section{Acknowledgements}

This study was funded by The Dutch National Care for the Elderly Program and sponsored by The Dutch Organization for Health Research and Development (ZonMw \#314070401). The funding source had no role in the study design, in the collection, analysis and interpretation of data, in the writing of the report or in the decision to submit the article for publication.

\section{Author details}

'Department of Health Services Research, Faculty of Health, Medicine and Life Sciences and CAHPRI School for Public Health and Primary care, Maastricht University, Maastricht, The Netherlands. ${ }^{2}$ Department of Family Medicine, Faculty of Health, Medicine and Life Sciences and CAHPRI School for Public Health and Primary care, Maastricht University, Maastricht, The Netherlands.

Received: 27 July 2015 Accepted: 5 January 2016

Published online: 12 January 2016

\section{References}

1. McCloskey R. Functional and self-efficacy changes of patients admitted to a Geriatric Rehabilitation Unit. J Adv Nurs. 2004;46(2):186-93.

2. Stucki G, Stier-Jarmer M, Grill E, Melvin J. Rationale and principles of early rehabilitation care after an acute injury or illness. Disabil Rehabil. 2005:27(7-8):353-9

3. Boston Working Group on Improving Health Care Outcomes Through Geriatric Rehabilitation. Med Care. 1997;35(6 Suppl):JS4-20.

4. Wells JL, Seabrook JA, Stolee P, Borrie MJ, Knoefel F. State of the art in geriatric rehabilitation. Part II: clinical challenges. Arch Phys Med Rehabil. 2003:84(6):898-903.

5. Bachmann S, Finger C, Huss A, Egger M, Stuck AE, Clough-Gorr KM. Inpatient rehabilitation specifically designed for geriatric patients: systematic review and meta-analysis of randomised controlled trials. BMJ. 2010;340:C1718

6. Kus S, Muller M, Strobl R, Grill E. Patient goals in post-acute geriatric rehabilitation-goal attainment is an indicator for improved functioning. J Rehabil Med. 2011:43(2):156-61.

7. Graham JE, Chang PF, Berges IM, Granger CV, Ottenbacher KJ. Race/ethnicity and outcomes following inpatient rehabilitation for hip fracture. J Gerontol A Biol Sci Med Sci. 2008;63(8):860-6.

8. Buijck BI, Zuidema SU, Spruit-van Eijk M, Bor H, Gerritsen DL, Koopmans RT. Is patient-grouping on basis of condition on admission indicative for discharge destination in geriatric stroke patients after rehabilitation in skilled nursing facilities? The results of a cluster analysis. BMC Health Serv Res. 2012:12:443.

9. Chang PF, Ostir GV, Kuo YF, Granger CV, Ottenbacher KJ. Ethnic differences in discharge destination among older patients with traumatic brain injury. Arch Phys Med Rehabil. 2008;89(2):231-6.

10. Chin $\mathrm{R}, \mathrm{Ng} \mathrm{B}$, Cheung LP. Factors predicting rehabilitation outcomes of elderly patients with hip fracture. Hong Kong Med J. 2008;14(3):209-15.
11. Brauer SG, Bew PG, Kuys SS, Lynch MR, Morrison G. Prediction of discharge destination after stroke using the motor assessment scale on admission: a prospective, multisite study. Arch Phys Med Rehabil. 2008;89(6):1061-5.

12. Boyd CM, Landefeld CS, Counsell SR, Palmer RM, Fortinsky RH, Kresevic D, et al. Recovery of activities of daily living in older adults after hospitalization for acute medical illness. J Am Geriatr Soc. 2008;56(12):2171-9.

13. Gill TM, Williams CS, Tinetti ME. The combined effects of baseline vulnerability and acute hospital events on the development of functional dependence among community-living older persons. J Gerontol A Biol Sci Med Sci. 1999;54(7):M377-83.

14. Vincent HK, Omli MR, Vincent KR. Absence of combined effects of anemia and bilateral surgical status on inpatient rehabilitation outcomes following total knee arthroplasty. Disabil Rehabil. 2010;32(3):207-15.

15. Meijer R, Ihnenfeldt DS, van Limbeek J, Vermeulen M, de Haan RJ. Prognostic factors in the subacute phase after stroke for the future residence after six months to one year. A systematic review of the literature. Clin Rehabil. 2003;17(5):512-20.

16. Meijer R, van Limbeek J, de Haan R. Development of the Stroke-unit Discharge Guideline: choice of assessment instruments for prediction in the subacute phase post-stroke. Int J Rehabil Res. 2006:29(1):1-8.

17. Spruit-van Eijk M, Zuidema SU, Buijck BI, Koopmans RT, Geurts AC. Determinants of rehabilitation outcome in geriatric patients admitted to skilled nursing facilities after stroke: a Dutch multi-centre cohort study. Age Ageing. 2012:41(6):746-52

18. Van Almenkerk S, Smalbrugge M, Depla MF, Eefsting JA, Hertogh CM. What predicts a poor outcome in older stroke survivors? A systematic review of the literature. Disabil Rehabil. 2013;35(21):1774-82.

19. Patrick L, Knoefel F, Gaskowski P, Rexroth D. Medical Comorbidity and Rehabilitation Efficiency in Geriatric Inpatients. J Am Geriatr Soc. 2001;49(11):1471-7

20. Hayden JA, Cote P, Bombardier C. Evaluation of the quality of prognosis studies in systematic reviews. Ann Intern Med. 2006;144(6):427-37.

21. Mols F, Vingerhoets AJ, Coebergh JW, van de Poll-Franse LV. Quality of life among long-term breast cancer survivors: a systematic review. Eur J Cancer. 2005:41(17):2613-9.

22. Kay E, Deutsch A, Chen D, Semik P, Rowles D. Effects of gender on inpatient rehabilitation outcomes in the elderly with incomplete paraplegia from nontraumatic spinal cord injury. J Spinal Cord Med. 2010;33(4):379-86.

23. New PW, Epi MC. Influence of age and gender on rehabilitation outcomes in nontraumatic spinal cord injury. J Spinal Cord Med. 2007;30(3):225-37.

24. Vincent HK, Vincent KR, Lee LW, Alfano AP. Effect of obesity on inpatient rehabilitation outcomes following total knee arthroplasty. Clin Rehabil. 2007;21(2):182-90.

25. Vincent KR, Vincent HK, Lee LW, Alfano AP. Inpatient rehabilitation outcomes in primary and revision total knee arthroplasty patients. Clin Orthop Relat Res. 2006:446:201-7.

26. Vincent KR, Vincent HK, Lee LW, Weng J, Alfano AP. Outcomes after inpatient rehabilitation of primary and revision total hip arthroplasty. Arch Phys Med Rehabil. 2006;87(8):1026-32

27. Sansone GR, Alba A, Frengley JD. Analysis of FIM instrument scores for patients admitted to an inpatient cardiac rehabilitation program. Arch Phys Med Rehabil. 2002;83(4):506-12.

28. Yan K, Pogoda T. Orthopaedic patient outcomes following interdisciplinary inpatient rehabilitation. Int J Ther Rehabil. 2013:20(7):361-6.

29. Berges IM, Kuo YF, Ostir GV, Granger CV, Graham JE, Ottenbacher KJ. Gender and ethnic differences in rehabilitation outcomes after hip-replacement surgery. Am J Phys Med Rehabil. 2008;87(7):567-72.

30. Hershkovitz A, Kalandariov Z, Hermush V, Weiss R, Brill S. Factors affecting short-term rehabilitation outcomes of disabled elderly patients with proximal hip fracture. Arch Phys Med Rehabil. 2007;88(7):916-21.

31. Kurichi JE, Ripley DC, Xie D, Kwong PL, Bates BE, Stineman MG. Factors associated with home discharge after rehabilitation among male veterans with lower extremity amputation. PM R. 2013:5(5):408-17.

32. Siebens HC, Sharkey P, Aronow HU, Horn SD, Munin MC, DeJong G, et al. Outcomes and weight-bearing status during rehabilitation after arthroplasty for hip fractures. PM R. 2012;4(8):548-55.

33. Vincent HK, Alfano AP, Lee L, Vincent KR. Sex and age effects on outcomes of total hip arthroplasty after inpatient rehabilitation. Arch Phys Med Rehabil. 2006:87(4):461-7.

34. Vincent KR, Vincent HK. A multicenter examination of the Center for Medicare Services eligibility criteria in total-joint arthroplasty. Am J Phys Med Rehabil. 2008;87(7):573-84. 
35. Vincent HK, DeJong G, Mascarenas D, Vincent KR. The effect of body mass index and hip abductor brace use on inpatient rehabilitation outcomes after total hip arthroplasty. Am J Phys Med Rehabil. 2009;88(3):201-9.

36. Field A. Discovering statistics using IBM SPSS statistics. 4th ed. Los Angeles: Sage; 2013.

37. Campbell SE, Seymour DG, Primrose WR, project ftAp. A systematic literature review of factors affecting outcome in older medical patients admitted to hospital. Age Ageing. 2004;33(2):110-5.

38. Carle AC. Mitigating systematic measurement error in comparative effectiveness research in heterogeneous populations. Med Care. 2010;48(6 Suppl):S68-74.

Submit your next manuscript to BioMed Central and we will help you at every step:

- We accept pre-submission inquiries

- Our selector tool helps you to find the most relevant journal

- We provide round the clock customer support

- Convenient online submission

- Thorough peer review

- Inclusion in PubMed and all major indexing services

- Maximum visibility for your research

Submit your manuscript at www.biomedcentral.com/submit
Biomed Central 